H. Urbach

S. Flacke

E. Keller

J.Textor

A. Berlis

A.Hartmann

J.Reul

L.Solymosi

H.H.Schild
H. Urbach ( $)$. S. Flacke · E. Keller · J. Textor - A. Berlis · J. Reul · H.H. Schild Department of Radiology/Neuroradiology, University Bonn, Sigmund-Freud-Str. 25, 53105 Bonn, Germany e-mail: urbach@uni-bonn.de

Tel.: + 49-228-287-6389

Fax: + 49-228-287-4321

\section{A. Hartmann}

Department of Neurology,

University Bonn, Sigmund-Freud-Str. 25,

53105 Bonn, Germany

\section{Solymosi}

Department of Neuroradiology,

University Würzburg,

Josef-Schneider-Str.11, 97080 Würzburg, Germany

\title{
Detectability and detection rate of acute cerebral hemisphere infarcts on CT and diffusion-weighted MRI
}

\begin{abstract}
Our purpose was to compare the detectability and detection rate of acute ischaemic cerebral hemisphere infarcts on CT and diffusion-weighted MRI (DWI). We investigated 32consecutive patients with acute hemisphere stroke with unenhanced CT and DWI within $6 \mathrm{~h}$ of stroke onset. The interval between CT and DWI ranged from 15 to $180 \mathrm{~min}$ (mean $60 \mathrm{~min}$ ). Infarct detectability on CT and DWI was determined by comparing the initial CT, DWI and later reference images in a consensus reading of five independent examiners. The "true" detection rate was assessed by analysing all single readings. Two patients had intracerebral haematomas on DWI and CT and were excluded. There were 27 patients with ischaemic infarcts; all were visible on DWI and proven by follow-up. DWI was negative in three patients without a final diagnosis of infarct (100\% sensitivity, $100 \%$ specificity, $\chi^{2}=30$, $P<0.0001)$. Ischaemic infarcts were
\end{abstract}

visible on 15 and not seen on $12 \mathrm{CT}$ studies (55\% sensitivity, $100 \%$ specificity, $\left.\chi^{2}=1.48, P=0.224\right)$. With regard to the single readings (30 examinations $\times 5$ ex-

aminers $=150$ readings), $63 \mathrm{CT}$ readings were true positive and 72 false negative (sensitivity $47 \%$, specificity $86 \%, \chi^{2}=2.88, P=0.089$ ).

Of the DWI readings 128 were true positive and 7 false negative (sensitivity $95 \%$, specificity $87 \%$, $\left.\chi^{2}=70.67, P<0.0001\right)$. Interobserver agreement was substantial for CT $(x=0.72,95 \%$ confidence interval, 0.6-0.84) and DWI $(x=0.82,95 \%$ confidence interval, 0.46-1). Taken together, detectability and detection rate of acute $(<6 \mathrm{~h})$ hemisphere infarcts are significantly higher with DWI than with CT.

Key words Brain, ischaemia . Magnetic resonance imaging, diffusion-weighted Computed tomography

\section{Introduction}

CT is considered the most important initial diagnostic study in patients with acute stroke [1]. It effectively discriminates between haemorrhagic and ischaemic stroke and signs of early ischaemia are often present within the first $3 \mathrm{~h}$ [2-9]. While in most thrombolysis studies, CT was used only to exclude intracerebral haemorrhage, in the ECASS I study CT criteria were established to exclude poor-outcome candidates before treatment [9-12].
Low density involving more than one third of the middle cerebral artery (MCA) territory was an exclusion criterion, but this sign was overlooked in 52of 62 patients, who were subsequently falsely enrolled in the study. Low density in more than one third of the MCA territory was highly predictive of a fatal outcome. Of 31 patients treated with recombinant tissue type plasminogen activator (rt-PA) who had this extent of low density on initial CT, 15 died within 7 days of admission, most from haemorrhage or space-occupying infarct oedema. If 
these patients had not been eliminated from the final analysis, a statistical difference between treated patients and those who received placebo would not have been found.

Diffusion-weighted MRI (DWI) has been shown to be exquisitely sensitive to acute cerebral ischaemia, and evaluation of the severity of ischaemia by means of the apparent diffusion coefficient (ADC) seems possible [13-15]. With echo-planar (EPI) acquisition DWI can be obtained within less than $1 \mathrm{~min}$, so that examination of patients with acute stroke becomes feasible. DWI has been shown to be more sensitive than conventional MRI to cerebral ischaemia but studies which compare CT and DWI are scarce [16-20]. Our aim was to evaluate the sensitivity of CT and DWI to early ischaemic infarcts. We therefore compared infarct detectability and "true" detection rate on CT and DWI in patients studied within $6 \mathrm{~h}$ of the onset of stroke.

\section{Methods}

Between October 1997 and November 1998 we studied 32consecutive patients with CT and DWI within $6 \mathrm{~h}$ after the onset of acute cerebral hemispheric symptoms. Two patients showing intracerebral haematomas on MRI were excluded; thus, 30 patients form the cohort of this retrospective study. The patients' clinical state was assessed as documented on admission, on repeated daily examinations and on re-examination at 90 days using the modified Rankin scale [21].

CT was obtained with a slice thickness of $4 \mathrm{~mm}$ infratentorially and $8 \mathrm{~mm}$ supratentorially. MRI was performed on a 1.5 Tesla system with a Powertrak 6000 gradient system (gradient strength $23 \mathrm{mT} / \mathrm{m}$, rise time $200 \mu \mathrm{s}$ ). Single- and multi-shot spin-echo (SE) EPI DWI sequences were performed (Table 1). DWI with three different gradient directions, and isotropic images were obtained Coronal 2D phase-contrast MR angiograms, axial T2-weighted GraSE, FLAIR-TSE and 3D-susceptibility-based perfusion sequences were performed in most patients, but not presented to the readers in this study. CT was performed in all patients within three days of the onset of stroke, and follow-up MRI in nine. Five neuroradiologists (EK, JR, AB, JT, LS), unblinded to the patients' symptoms, independently studied the initial CT studies and were asked to draw the tissue they thought to be infarcted on brain slice diagrams from a neuroanatomical/neuroimaging atlas [22]. On different days they were asked to draw the tissue they assessed to be infarcted on DWI on similar diagrams. Infarct detectability on CT and DWI and the definite extent of the infarct on follow-up CT or MRI were determined by a consensus panel of all readers. In addition, the "true" infarct detection rate was determined by analysing all single CT and DWI readings. CT and DWI readings were judged as "true positive" if an ischaemic infarct as proven by follow-up CT or MRI was correctly described, and as assessed by the consensus panel. A correctly localised infarct which was under- or overestimated in size was also judged "true positive". If an infarct was not confirmed on follow-up, the readings were judged "false positive". CT and DWI readings were judged "true negative" if no early infarcts were detected, and this was confirmed by follow-up CT or MRI. They were defined as "false negative" if early infarcts were visible on the complementary images and follow-up CT or MRI.
Table 1 Parameters for spin-echo echo-planar diffusion-weighted imaging (DWI)

\begin{tabular}{lll}
\hline & Single-shot & Multi-shot \\
\hline Repetition time $(\mathrm{ms})$ & 5000 & 1500 \\
Echo time $(\mathrm{ms})$ & 120 & 105 \\
Diffusion time $(\mathrm{ms})$ & 85 & 85 \\
Flip angle $\left(^{\circ}\right)$ & 90 & 90 \\
Field of view $(\mathrm{cm} \times \mathrm{cm})$ & $16 \times 23$ & $16 \times 23$ \\
Matrix & $92 \times 128$ & $102 \times 128$ \\
Half-Fourier factor & 0.603 & 0.642 \\
Orientation & axial & axial \\
Slices & 20 & 12 \\
Slice thickness and gap $(\mathrm{mm})$ & 6,1 & 6,3 \\
Echo-planar factor & 63 & 23 \\
b $\left(10^{-5} / \mathrm{mm}^{2}\right)$ & 0,800 & $0,500,1000$ \\
Number of Excitations & 1 & 3 \\
Acquisition time $(\mathrm{min})$ & 0.30 & 3.18 \\
\hline
\end{tabular}

Sensitivity and specificity were calculated for CT and DWI examinations and for the readings. A $\chi^{2}$ test was used to assess detectability and detection rate of CT and DWI, respectively. Paired $x$ coefficients were calculated for all five examiners. In addition, a multiple observer $\varkappa$ coefficient with standard error was calculated for dichotomous ratings for all five examiners combined [23]. Approximate $95 \%$ confidence intervals were calculated from $\varkappa \pm 2 \times$ standard error.

\section{Results}

We studied 18 men and 12 women (18-76 years, mean 52years). CT was the initial examination in 25 patients, MRI in five patients. The interval between CT and DWI ranged from 15 to $180 \mathrm{~min}$ (mean $60 \mathrm{~min}$ ). Ten patients had mild or even resolving neurological deficits on admission and 20 had major neurological deficits. There were 16 patients with a favourable outcome at 90 days (Rankin 0-2), nine severely disabled (Rankin 3-5), and five who died within the first 7 days.

Interpretation of $15 \mathrm{CT}$ examinations was "true positive", three "true negative". No CT examination was "false positive" but 12 were "false negative" (55\% sensitivity, $100 \%$ specificity, $\chi^{2}=1.48, P=0.224$ ) (Tables 2 , $3)$. Of the DWI examinations 27 were "true positive" and three "true negative". There were no "false negative" or "false positive" examinations (100\% sensitivity, $100 \%$ specificity, $\left.\chi^{2}=30, P<0.0001\right)$.

Of the $150 \mathrm{CT}$ readings, 52were "true positive", three "false positive". In nine readings, the infarct was smaller and in two it was larger than on DWI and follow-up examintions; for statistical evaluation, these readings were taken as "true positive". There were 12 "true negative" and 72 "false negative" readings $(47 \%$ sensitivity, $86 \%$ specificity, $\chi^{2}=2.88, P=0.089$ ). Of the 150 DWI readings, 128 were "true positive", two "false positive", 13 "true negative" and seven "false negative" $\left(95 \% \quad\right.$ sensitivity, $\quad 87 \% \quad$ specificity, $\quad \chi^{2}=70.67$, 
Table 2 Patient data and imaging findings on CT and DWI

\begin{tabular}{|c|c|c|c|c|c|c|}
\hline Patient & $\begin{array}{l}\text { Age (years) } \\
\text { sex }\end{array}$ & Site of infarct ${ }^{\mathrm{a}}$ & Rankin score $^{\mathrm{b}}$ & $\begin{array}{l}\text { Consensus } \\
\text { CT/DWI }\end{array}$ & CT readings ${ }^{\mathrm{d}}$ & $\begin{array}{l}\text { DWI } \\
\text { readings }\end{array}$ \\
\hline 1 & 67 , male & Entire middle cerebral artery territory & 6 & $-1+$ & ----- & +++++ \\
\hline $271, \mathrm{~m}$ & & $\begin{array}{ll}\text { Entire hemisphere } & 6\end{array}$ & $-1+$ & & - & \\
\hline 3 & 55 , male & Entire middle cerebral artery territory & 6 & $+/+$ & $-\mathrm{u}-+\mathrm{u}$ & +++++ \\
\hline 4 & 59 , male & Striatocapsular, temporoparietal & 6 & $+/+$ & uuuuu & +++++ \\
\hline 5 & 63 , male & Entire middle cerebral artery territory & 6 & $+/+$ & +++++ & +++++ \\
\hline 6 & 48 , male & Frontal, central & 4 & $+/+$ & +-+++ & +++++ \\
\hline 7 & 74 , male & Striatocapsular, frontal & 4 & $+/+$ & +++++ & +++++ \\
\hline 8 & 72, male & Striatocapsular, frontal & 4 & $+/+$ & +++++ & +++++ \\
\hline 9 & 50 , female & Striatocapsular & 3 & $-1+$ & ----- & +++++ \\
\hline 10 & 72 , male & Striatocapsular & 3 & $-1+$ & ----- & +++++ \\
\hline 11 & 62, male & Striatocapsular & 3 & $-1+$ & ----- & +++++ \\
\hline 1239 , fer & male $\quad \mathrm{F}$ & Peripheral MCA 3 & $-1+$ & & ++ & \\
\hline 13 & 37, female & Striatocapsular, peripheral MCA & 3 & $+/+$ & $+\mathrm{u}++\mathrm{u}$ & +++++ \\
\hline 14 & 39 , male & Frontal & 3 & $+/+$ & +++++ & +++++ \\
\hline 15 & 37 , male & Striatocapsular, frontal & $2+/+$ & +++++ & +++++ & \\
\hline 16 & 63 , male & Posterior cerebral artery territory & $2+/+$ & +++++ & +++++ & \\
\hline 17 & 34 , male & Insula & 1 & $-/+$ & ----- & +++++ \\
\hline 18 & 66 , male & Precentral gyrus & 1 & $-1+$ & ----- & +++++ \\
\hline 19 & 76 , male & Insula & 1 & $-1+$ & ----- & +++++ \\
\hline 20 & 48 , male & Anterior choroidal artery territory & 1 & $-1+$ & ----- & +++++ \\
\hline 21 & 73 , female & Posterior cerebral artery territory & 1 & $-/+$ & ----- & +-+-+ \\
\hline 22 & 57 , male & Anterior cerebral artery territory & 1 & $+/+$ & $-\mathrm{w}--+$ & +++++ \\
\hline 23 & 32 , female & Frontal operculum & 1 & $+/+$ & -+-++ & +++++ \\
\hline 24 & 28 , female & Frontal, central MCA & 1 & $+/+$ & -++-+ & +++++ \\
\hline 25 & 53 , male & Insula & 1 & $+/+$ & ++-++ & +++++ \\
\hline 26 & 18 , female & Striatocapsular & 0 & $+/+$ & $++\mathrm{OO}+$ & +++++ \\
\hline 27 & 68 , female & Precentral gyrus & 0 & $-/+$ & ----- & $-\mathrm{W}---$ \\
\hline 28 & 51 , female & No infarct & 0 & $-1-$ & ----- & ----- \\
\hline 29 & 49 , female & No infarct & 0 & $-1-$ & $-\mathrm{f}---$ & ----- \\
\hline 30 & 31 , female & No infarct & 0 & $-1-$ & $-\mathrm{ff}--$ & ff--- \\
\hline
\end{tabular}

Table 3 Detectability and detection rates of infarcts on CT and diffusion-weighted imaging (DWI)

\begin{tabular}{lll}
\hline & CT & DWI \\
\hline Detectability (30 cases) & & \\
Sensitivity (\%) & 55 & 100 \\
Specificity (\%) & 100 & 100 \\
$\chi^{2}$ & 1.48 & 30.00 \\
$P$ & 0.224 & $<0.0001$ \\
Detection rate (30 cases, 5 readers) & & \\
Sensitivity (\%) & 47 & 95 \\
Specificity (\%) & 86 & 87 \\
$\chi^{2}$ & 2.88 & 70.67 \\
$P$ & 0.089 & $<0.0001$ \\
$\varkappa$ & 0.720 .82 & \\
Standard error of mean & 0.06 & 0.18 \\
\hline
\end{tabular}

$P<0.0001)$. Isotropic DWI without major motion artefacts were obtained in 23 patients. In seven others the readers had to rely on anisotropic images; in these cases, single-shot images were of better diagnostic quality. On the seven "false negative" DWI readings, a small cortical MCA territory infarct was not seen due to motion artefact and a small posterior cerebral artery territory infarct was overlooked by two examiners. In two "false positive" readings, diffusion anisotropy of the internal capsule was misinterpreted as an ischaemic lesion.

$\varkappa$ analysis revealed substantial agreement among the five examiners for both CT and DWI. Paired $\varkappa$ coefficients ranged from 0.72 to 0.87 for $\mathrm{CT}$ and from 0.87 to 1 for DWI. The multiple observer $\varkappa$ coefficient was $0.72(95 \%$ confidence interval, $0.6-0.84)$ for CT and $0.82(95 \%$ confidence interval, $0.46-1)$ for DWI. The higher interobserver agreement on DWI was explained in part by six false CT readings, in which different examiners thought brain parenchyma was infarcted which proved to be normal on follow-up. In three readings the final diagnosis was "no infarct"; in one, an infarct was visible in a different location. In two readings a striatocapsular infarct was correctly diagnosed, but a cortical MCA territory infarct was not confirmed on follow-up. 
Fig.1 a A right anterior cerebral artery territory infarct was not seen on CT by four of five examiners. b A single-shot diffusion-weighted image (DWI) shows the infarct despite motion artefacts
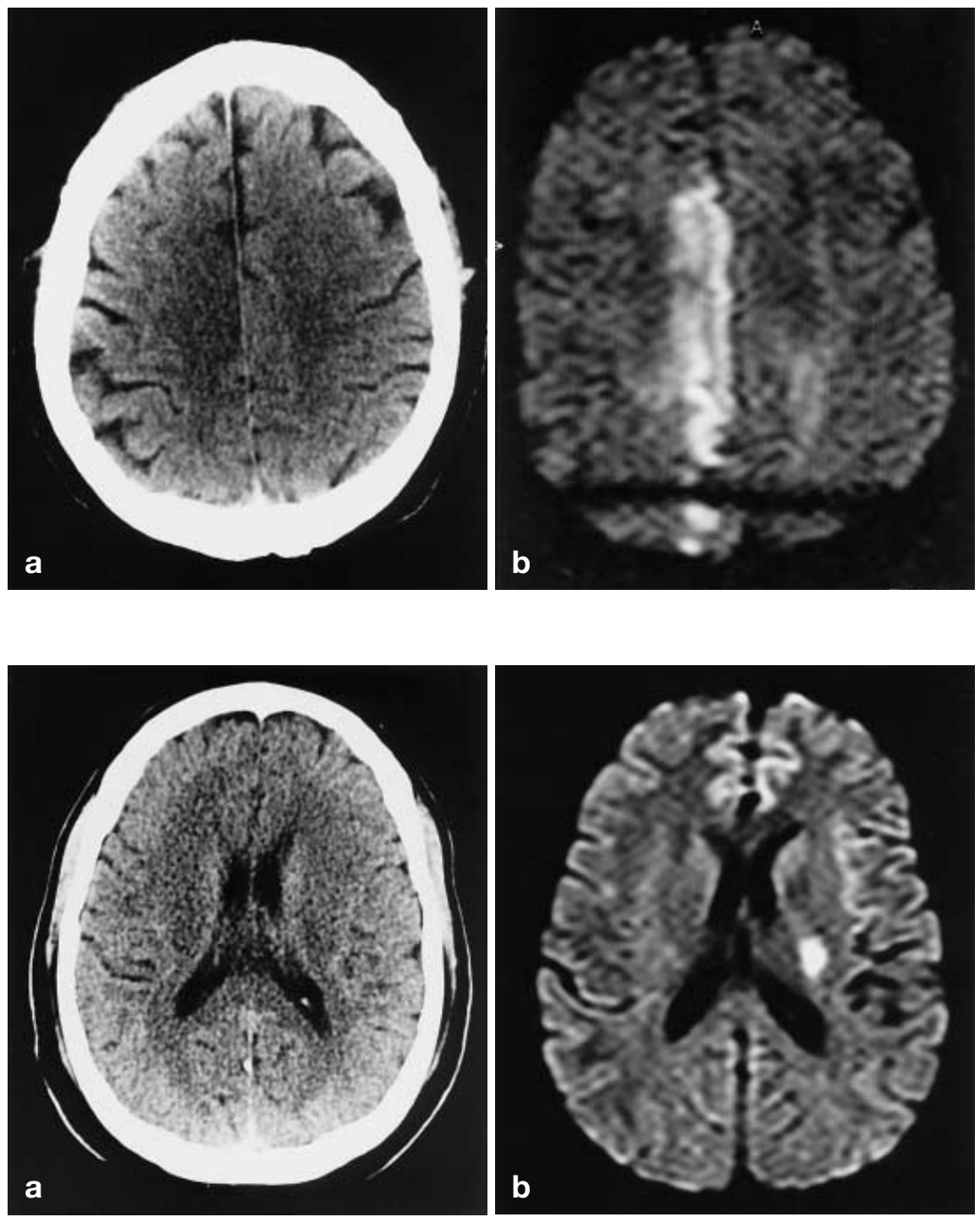

Fig. 2 a CT 115 min after the onset of a right hemiparesis shows no clear low-density lesion. b Multishot DWI $35 \mathrm{~min}$ later shows a left anterior choroidal artery territory infarct
In the nine patients who had low density in more than one third of the MCA territory on follow-up CT, the lesion was correctly localised on CT in 23 and underestimated in seven of 45 readings ( $66 \%$ sensitivity). In 15 of these readings a lesion was correctly localised on DWI, but not seen on CT.

\section{Discussion}

In acute stroke, CT is used to exclude intracranial haemorrhage and other conditions which can mimic a stroke and to show signs of early ischaemia. Several studies of early ischaemia report parenchymal low density as the most frequent finding [2-9]. It typically represents irreversibly damaged tissue [6] and is mainly caused by increased tissue water [24]. In experimental brain oedema, there is an increase in water of $2 \%$ wet weight after $2 \mathrm{~h}$ and $2.9 \%$ after $4 \mathrm{~h}$; this is accompanied by a parallel increase in sodium and decrease in potassium [25]. Experimental studies suggest a change of 2.6 Hounsfield units (HU) per $1 \%$ change in water content [26]. Histographic analysis of the density values of each pixel confirms the early changes of ischaemia occurring, in a small range of 5-10 HU [8]. The ability of the eye to differentiate grey shades is limited to $15-20$ steps, so that in a normal window range of $90 \mathrm{HU}$ there is a change of around 2grey shades within that early period. These slight density changes may well be overlooked on early CT, but can be highlighted by postprocessing, which indicates the difficulty in seeing and quantifying the early infarct [8].

Our aim was to compare the sensitivity of CT and DWI to early ischaemic infarcts. Since we compared two modalities carried out in the same time range we 
were able to distinguish between infarct detectability and detection rate. In prior CT studies, it was difficult to distinguish between detectability and detection rate, since one could not decide whether an infarct was not present at the time of the initial examination or not yet visible. It was possible to decide that an infarct was present, but not visible, if it was seen on the complementary examination and confirmed by follow-up. Infarct detectability was determined in a consensus reading of all five examiners, who compared the initial CT, DWI and the follow-up images. The "true" infarct detection rate was analysed by comparing the independent single CT and DWI readings with the consensus readings.

In prior studies, infarct detectability and detection rate were not distinguished, and the "sensitivity" of CT to parenchymal low density in the first $6 \mathrm{~h}$ after the onset of symptoms ranged from 56 to $95 \%[2-9,27]$. This "sensitivity" depends, among other things, on the duration and severity of ischaemia, the size, type and location of the infarct and the examiner's experience [6-7]. For example, in patients with MCA trunk occlusions, overall sensitivity was about $80 \%$ and increased asymptotically to $100 \%$ within $3 \mathrm{~h} \mathrm{[6].} \mathrm{When} \mathrm{a} \mathrm{less} \mathrm{selected} \mathrm{cohort} \mathrm{of}$ patients was studied within $6 \mathrm{~h}$, sensitivity was only $66 \%$, and $28 \%$ of the patients had "false negative" initial CT [27]. In our study, infarct detectability on CT was lower than in most previous studies. This is probably explained by there being a high number of small cortical infarcts: ten cortical or striatocapsular infarcts less than $2 \mathrm{~cm}$ in diameter were not seen on CT but visible on DWI; in one of these cases DWI was performed prior to CT. Only two infarcts involving more than two thirds of the MCA territory were missed on CT and visible on DWI. These infarcts were studied later with DWI, and might have been visible on CT at that time. However, even when these examinations are excluded from the analysis, the sensitivity of CT only rises up to $60 \%$, still far below that of DWI.

Interobserver agreement was substantial for both CT and DWI. The higher agreement rate on DWI can be in part explained by a small number of CT readings ( 6 of 150 ), in which the examiners assessed as infarcted brain parenchyma which proved to be normal on follow-up. In three readings the final diagnosis was "no infarct". In one reading an infarct was in an unaffected area. In two readings striatocapsular involvement was correctly diagnosed, but the cortical MCA territory falsely assessed to be infarcted. These false readings presumably occurred because the examiners knew the symptoms and overinterpreted the initial CT as showing subtle signs of early ischaemia. Interobserver agreement was substantial among neuroradiologists having various degrees of experience; this argues against the notion that detection of acute hemisphere infarcts is mainly dependent on the examiner's experience.

DWI has generated great interest since the demonstration that substantial changes in parenchymal diffu- sion of water are rapidly detectable after the onset of cerebral ischaemia [13]. DWI shows areas of reduced diffusion before substantial changes in water content occur [28]. Cytotoxic oedema, increased cytosolic viscosity, and increased tortuoisity of the extracellular space may cause a decrease in the diffusion coefficient; the exact mechanisms are still the subject of research [29-30]. In animal experiments, regions with reduced parenchymal diffusion of water were detected within $5 \mathrm{~min}$ of the onset of ischaemia $[13,15]$. Some early, less severe diffusion abnormalities were potentially reversible [14]. In human studies, it is more difficult to determine the sensitivity of DWI as the examinations are usually obtained at a later, single point in time. It is still unknown whether DWI abnormalities can be reversible. In our study, a high-signal DWI lesion invariably progressed to a high-signal lesion on $\mathrm{T} 2$-weighted images or a low-density lesion on follow-up CT.

DWI shows areas of reduced diffusion as high signal. Potential pitfalls are effects of diffusion anisotropy, the amount of regional high signal on images without diffusion weighting and artefacts caused by susceptibility, especially when echo-planar acquisition is used [31]. Anisotropy effects are minimised by using isotropic images. In practice, so-called diffusion trace images are obtained by averaging images obtained with diffusion gradients applied in three orthogonal planes [29]. However, when head movements affect one of the three acquisitions, calculation of diffusion trace images becomes impossible and image interpretation has to rely on one of the unaveraged images. Thus, the clinician has to be familiar with anisotropy effects to avoid false interpretation. In our study all 27 infarcts were visible on the initial DWI. Two "false positive" and seven "false negative" readings occurred, however, due to motion artefacts or to misinterpretation of anisotropy effects. Motion artefacts are likely to occur in patients with an acute stroke, who are often in poor condition. We therefore included single- and multishot DWI in our protocol, because single-shot images are less sensitive to motion artefacts but have a lower signal-to-noise ratio [32]. Despite the use of single-shot DWI, one small cortical infarct was not seen due to motion artefacts, and one infarct was overlooked by two examiners. Both infarcts, however, would have been diagnosed when perfusion images would have been evaluated.

A limitation of this study was the time between CT and DWI examinations. For several reasons we do not consider this too damaging. In five patients initially studied with DWI and three in whom DWI was performed within 45 min of CT, we found three striatocapsular and small cortical infarcts visible only on DWI. Visible cortical infarcts were overlooked on CT following DWI (17 of 45 readings). In three readings, infarcts were overlooked on CT performed 215-360 min after stroke onset. 


\section{References}

1. Adams HP, Brott TG, Furlan AJ, et al (1996) Guidelines for thrombolytic therapy for acute stroke: a supplement for the management of patients with acute ischemic stroke. Stroke 27: 1711-1718

2. Bozzao I, Bastianello S, Fantozzi LM, Angeloni U, Argentino C, Fieschi C (1988) Correlation of angiographic and sequential CT findings in patients with evolving cerebral infarction. AJNR 10: 1215-1222

3. Tomura N, Uemura K, Inugami A, Fujita H, Higano S, Shishido F (1988)

Early CT findings in cerebral infarction. Radiology 168: 463-467

4. Truwit CL, Barkovich AJ, Gean-Marton A, Hibri N, Norman D (1990) Loss of the insular ribbon: another early sign of acute middle cerebral artery infarction. Radiology 176: 801-806

5. Horowitz SH, Zito JL, Donnarumma R, Patel M, Alvir J (1991) Computed tomographic-angiographic findings within the first five hours of cerebral infarction. Stroke 22: 1245-1253

6. Von Kummer R, Meyding-Lamade U, Forsting M, et al (1994) Sensitivity and prognostic value of early CT in occlusion of the middle cerebral artery trunk. AJNR 15: 9-15

7. Urbach H, Klotz S, Solymosi L (1996) Detectability of acute supratentorial brain infarctions by computed tomography. Akt Neurol 23: 146-150

8. Bendszus M, Urbach H, Meyer B, Schultheiß R, Solymosi L (1997) Improved CT diagnosis of acute middle cerebral artery territory infarctions with the density-difference analysis. Neuroradiology 39: 127-131

9. Von Kummer R, Allen KL, Holle R, et al (1997) Acute stroke: usefulness of early CT findings before thrombolytic therapy. Radiology 205: 327-333

10. The National Institute of Neurological Disorders and Stroke rt-PA Stroke Study Group (1995) Tissue plasminogen activator for acute ischemic stroke. N Engl J Med 333: 1581-1587

11. Del Zoppo GJ, Higashida RT, Furlan AJ, Pessin MS, Rowley HA, Gent M, and the PROACT investigators (1998) PROACT: a phase II randomized trial of recombinant pro-urokinase by direct arterial delivery in acute middle cerebral artery stroke. Stroke 29: 4-11
12. Hacke W, Kaste M, Fieschi C, et al (1995) Intravenous thrombolysis with recombinant tissue plasminogen activator for acute hemispheric stroke. JAMA 274: 1017-1025

13. Moseley ME, Cohen Y, Mintorovitch J, et al (1990) Early detection of regional cerebral ischemia in cats: comparison of diffusion- and T2-weighted MRI and spectroscopy. Magn Reson Med 14: 330-346

14. Hasegawa Y, Fisher M, Latour LL, Dardzinski BJ, Sotak CH (1994) MRI diffusion mapping of reversible and irreversible ischemic injury in focal brain ischemia. Neurology 44: 1484-1490

15. Reith W, Hasegawa Y, Latour LL, Dardzinski BJ, Sotak CH, Fisher M (1995) Multislice diffusion mapping for $3-\mathrm{D}$ evolution of cerebral ischemia in a rat stroke model. Neurology 45: 172-177

16. Warach S, Dashe JF, Edelman RR (1996) Clinical outcome in ischemic stroke predicted by early diffusionweighted and perfusion magnetic resonance imaging: a preliminary analysis. J Cereb Blood Flow Metab 16: 53-59

17. Sorensen A, Buonanno F, Gonzalez RG, et al (1996) Hyperacute stroke: Evaluation with combined multisection diffusion-weighted and hemodynamically weighted echo-planar MR imaging. Radiology 199: 391-401

18. Flacke S, Keller E, Hartmann A, et al (1998) Improved diagnosis of early cerebral infarct by the combined use of diffusion and perfusion imaging. Fortschr Röntgenstr 168: 493-501

19. Van Everdingen KJ, van der Grond J, Kappelle LJ, Ramos LMP, Mali WPTM (1998) Diffusion-weighted magnetic resonance imaging in acute stroke. Stroke 29: $1783-1790$

20. Gonzalez RG, Schaefer PW, Buonanno FS, et al (1999) Diffusion-weighted MR imaging: diagnostic accuracy in patients imaged within 6 hours of stroke symptom onset. Radiology 210: 155-162

21. Van Switen JC, Koudstaal PJ, Visser MC, Shouten HJA, Van Gijn J (1988) Interobserver agreement for the assessment of handicap in stroke. Stroke 19: 604-607

22. Kretschmann H, Weinrich W (1992) Cranial neuroimaging and clinical neuroanatomy. Thieme, Stuttgart, pp 86-117
23. Fleiss JL (1971) Measuring nominal scale agreement among many raters. Psychol Bull 76: 378-382

24. Torack RM, Alcada H, Gado M (1975) Water, specific gravity and histology as determinations of diagnostic computerized cranial tomography (CCT). In: Pappius HM, Feindel W (eds) Dynamics of brain edema. Springer, Berlin Heidelberg New York, pp 271-278

25. Schuier FJ, Hossmann KA (1980) Experimental brain infarcts in cats. II. Ischemic brain edema. Stroke 6: 593-602

26. Unger E, Littlefield J, Gado M (1988) Water content and water structure in CT and MR signal changes: possible influence in detection of early stroke. AJNR 9: 687-691

27. Bourquain H, Elsner E, Gerber J, Müller A, Straube T, von Kummer R (1998) Prospektiver Wert der frühen CT bei zerebraler Ischämie. Klin Neuroradiol 8: 135-136

28. Mintorovitch J, Yang GY, Shimizu H, Kucharczyk J, Chan PH, Weinstein PR (1994) Diffusion-weighted magnetic resonance imaging of acute focal cerebral ischemia: comparison of signal intensity with changes in brain water and $\mathrm{Na}+$, $\mathrm{K}(+)$-ATPase activity. J Cereb Blood Flow Metab 14: 332-336

29. Baird AE, Warach S (1998) Magnetic resonance imaging of acute stroke. J Cereb Blood Flow Metab 18: 583-609

30. Van der Toorn A, Dijkhuizen RM, Tulleken CA, Nicolay K (1996) Diffusion of metabolites in normal and ischemic rat brain measured by localized $1 \mathrm{H}$ MRS. Magn Reson Med 36: 914-922

31. Warach S, Boska M, Welch KMA (1997) Pitfalls and potential of clinical diffusion-weighted MR imaging in acute stroke. Stroke 28: 481-482

32. Mürtz P, Flacke S, Träber F, et al (1998) Diffusion-weighted MR imaging: navigated multi-shot SE EPI technique for clinical use. Fortschr Röntgenstr 168: 580-588

33. Baird AE, Benfield A, Schlaug G, et al (1997) Enlargement of human cerebral ischemic lesion volumes measured by diffusion-weighted magnetic resonance imaging. Ann Neurol 41: 581-589

34. Rordorf G, Koroshetz WJ, Copen WA, et al (1998) Regional ischemia and ischemic injury in patients with acute middle cerebral artery stroke as defined by early diffusion-weighted and perfusion-weighted MRI. Stroke 29: 939-943 